Marquette University

e-Publications@Marquette

Chemistry Faculty Research and Publications

Chemistry, Department of

$1-1-2016$

\title{
On Stabilization of Scattering Resonances in Recombination Reaction that Forms Ozone
}

Mikhail V. Ivanov

Marquette University

Dmitri Babikov

Marquette University, dmitri.babikov@marquette.edu

Published version. Journal of Chemical Physics, Vol. 144, No. 15 (2016). DOI. (C) 2016 American Institute of Physics. Used with permission. 


\title{
On stabilization of scattering resonances in recombination reaction that forms ozone
}

\author{
Mikhail V. Ivanov and Dmitri Babikova) \\ Chemistry Department, Wehr Chemistry Building, Marquette University, Milwaukee, \\ Wisconsin 53201-1881, USA
}

(Received 16 February 2016; accepted 29 March 2016; published online 15 April 2016)

\begin{abstract}
Calculations of energy transfer in the recombination reaction that forms ozone are carried out within the framework of the mixed quantum/classical theory and using the dimensionally reduced 2D-model of ozone molecule, with bending motion neglected. Recombination rate coefficients are obtained at room temperature for symmetric and asymmetric isotopomers of singly and doubly substituted isotopologues. The processes of resonance formation, spontaneous decay, collisional dissociation, and stabilization by bath gas (Ar) are all characterized and taken into account within the steady-state approximation for kinetics. The focus is on stabilization step, where the mysterious isotopic $\eta$-effect was thought to originate from. Our results indicate no difference in cross sections for stabilization of scattering resonances in symmetric and asymmetric isotopomers. As practical results, the general and simple analytic models for stabilization and dissociation cross sections are presented, which can be applied to resonances in any ozone molecule, symmetric or asymmetric, singly or doubly substituted. Present calculations show some isotope effect that looks similar to the experimentally observed $\eta$-effect, and the origin of this phenomenon is in the rates of formation/decay of scattering resonances, determined by their widths, that are somewhat larger in asymmetric isotopomers than in their symmetric analogues. However, the approximate two-dimensional model used here is insufficient for consistent and reliable description of all features of the isotopic effect in ozone. Calculations using an accurate 3D model are still needed. Published by AIP Publishing. [http://dx.doi.org/10.1063/1.4945779]
\end{abstract}

\section{INTRODUCTION}

Anomalous enrichment of stratospheric ozone in heavy isotope ${ }^{18} \mathrm{O}^{1,2}$ and the mass-independent fractionation (MIF) of oxygen isotopes in the laboratory ozone experiments $\mathrm{s}^{3,4}$ were discovered more than 30 years ago. Over this period of time many geoscientists and physical chemists in both experimental and theoretical communities tried to elucidate the molecular level origin of these phenomena, but despite any substantial progress made on the topic, several gray areas still remain. ${ }^{5-8}$

It was established experimentally that the MIF of oxygen occurs due to large and unusual isotope effect in the recombination reaction that forms ozone

$$
\begin{gathered}
\mathrm{O}_{2}+\mathrm{O} \rightarrow \mathrm{O}_{3}^{*}, \\
\mathrm{O}_{3}^{*}+\mathrm{M} \rightarrow \mathrm{O}_{3}+\mathrm{M}^{*} .
\end{gathered}
$$

Here $\mathrm{M}$ denotes a quencher (can be any atmospheric molecule, or $\mathrm{Ar}$ atom in the laboratory experiments) whose role is to carry away energy released by the metastable intermediate $\mathrm{O}_{3}^{*}$ (scattering resonance, excited rotationally and vibrationally above dissociation threshold). Experimental studies of various isotopic substitutions in this reaction indicate that asymmetric ozone molecules are formed at higher rates, compared to their symmetric isotopomers. ${ }^{9-14}$ For example, consider ozone molecule substituted with one ${ }^{18} \mathrm{O}$ atom, a singly substituted

\footnotetext{
a) Author to whom correspondence should be addressed. Electronic mail: dmitri.babikov@mu.edu
}

isotopologue. It has two possible isotopomers: asymmetric ${ }^{16} \mathrm{O}^{16} \mathrm{O}^{18} \mathrm{O}$ and symmetric ${ }^{16} \mathrm{O}^{18} \mathrm{O}^{16} \mathrm{O}$. Experimental results indicate ${ }^{13}$ that for asymmetric ${ }^{16} \mathrm{O}^{16} \mathrm{O}^{18} \mathrm{O}$ the total rate of formation is $10 \%$ higher than the rate of formation of symmetric ${ }^{16} \mathrm{O}^{18} \mathrm{O}^{16} \mathrm{O}$. In Earth's atmosphere this singly substituted isotopologue is most abundant (besides the standard ozone), so understanding the origin of this phenomenon has paramount importance for interpretation of the MIF of oxygen in general. A similar effect occurs in the doubly substituted isotopologue of ozone (rare in the atmosphere, but readily produced in the laboratory experiments to obtain more insight into the process). Namely, for asymmetric isotopomer ${ }^{16} \mathrm{O}^{18} \mathrm{O}^{18} \mathrm{O}$, the total rate of formation is almost $16 \%$ higher compared to the formation rate of symmetric isotopomer ${ }^{18} \mathrm{O}^{16} \mathrm{O}^{18} \mathrm{O} \cdot{ }^{13}$ Overall, all symmetric ozone molecules (five different isotopologues, see Refs. 13 and 14) exhibit very similar formation rates that are about $20 \%$ lower than the general trend of asymmetric isotopomers. ${ }^{13}$

Rigorous theoretical explanation of this phenomenon is still missing. In early 2000's, in order to incorporate this feature into their statistical model of ozone formation, Gao and Marcus introduced $\eta$-effect empirically, ${ }^{15-17}$ based on experimental evidence. They hypothesized that this nonstatistical effect may occur during the stabilization step of the recombination reaction, process (2), and argued that in symmetric molecules the density of dynamically active states may be lower, compared to asymmetric molecules. ${ }^{15}$ Marcus also discussed several other alternative sources of $\eta$-effect, such as spin-orbit coupling ${ }^{18}$ in the asymptotic region of the 
potential energy surface (PES), resonance coupling effect, ${ }^{19}$ chaotic behavior of trajectories at high energies ${ }^{18}$ and, more recently, the Coriolis coupling effect ${ }^{20,21}$ that may be different in symmetric and asymmetric isotopomers of $\mathrm{O}_{3}$. While all these are legitimate hypotheses, no convincing proof has ever been shown for any of these.

Instead of postulating the effect and then trying to find a suitable explanation for it, it would be more valuable to develop a rigorous theoretical description for the recombination process, based on first principles. Thus, in 2000s several groups proposed quantum dynamical treatments of the process (2), using standard methods of inelastic scattering, such as time independent coupled-channel formalism. In order to make these full-quantum calculations computationally affordable, Charlo and Clary ${ }^{22,23}$ employed a sudden-collision assumption for $\mathrm{O}_{3}^{*}+\mathrm{Ar}$ encounter, and a frozen-bending approximation for $\mathrm{O}_{3}^{*}$ vibrations. Unfortunately, their groundbreaking work suffered from the use of Jacobi coordinates for description of the multi-channel process and was not particularly successful in reproducing the isotope effect. The next study by Xie and Bowman ${ }^{24}$ offered a considerable improvement by implementing the full-dimensional treatment of $\mathrm{O}_{3}^{*}$. However, their calculations have been carried out for very few (just three) combinations of impact parameter of $\mathrm{Ar}$ and orientation of $\mathrm{O}_{3}^{*}$. Ivanov and Schinke ${ }^{25}$ reported calculations of state-to-state transitions that were converged with respect to the number of partial waves, but their basis set included just the bound states of $\mathrm{O}_{3}$, and no scattering resonances $\mathrm{O}_{3}^{*}$. Notably, no isotope effects due to symmetry were found in any of these calculations. Origin of the mysterious $\eta$-effect remained unidentified.

One recognizes that the exact quantum calculations are prohibitively expensive for the process (2) by looking at the work done by the groups of Clary, ${ }^{22,23}$ Bowman, ${ }^{24}$ and Schinke. ${ }^{25}$ They all used the sudden collision approximation and carried out calculations for non-rotating ozone molecule $(J=0)$ only, and also each of them employed at least one more simplifying assumption in addition to the first two. Neither incorporated lifetimes of $\mathrm{O}_{3}^{*}$ into their treatments of kinetics.

More recently, in early 2010s, ${ }^{26-30}$ we proposed an alternative method for theoretical treatment of the process (2) - a mixed quantum/classical theory for collisional energy transfer and ro-vibrational energy flow. Our method is still approximate, but it allows bypassing some of the difficulties described above. First of all, our method allows avoiding the sudden collision approximation and treating the dynamics of $\mathrm{O}_{3}^{*}+\mathrm{Ar}$ scattering in a time-dependent manner (classically). This is important, since a typical $\mathrm{O}_{3}^{*}+\mathrm{Ar}$ collision is not sudden, but is more like a slow adiabatic process, where the fast vibrational motion of $\mathrm{O}_{3}^{*}$ is able to adjust continuously to position of Ar atom during the scattering process. Second, we incorporate the rotation of $\mathrm{O}_{3}^{*}$ including a broad range of rotational excitations, up to $J \sim 90$ and compute the rotational quenching of $\mathrm{O}_{3}^{*}$ in collisions with $\mathrm{Ar}$ (also classically). These classical approximations are justified because, in this reaction, we do not expect any quantum effects due to scattering of heavy $\mathrm{Ar}$ or due to rotation of heavy $\mathrm{O}_{3}^{*}$. However, quantum mechanics is retained for description of the vibrational motion in $\mathrm{O}_{3}^{*}$, which incorporates zero-point energy and symmetry of vibrational modes, quantization of states, scattering resonances, including accurate calculations of their formation/decay rates and their collision-induced dissociation.

In this paper we apply an improved version of our mixed quantum/classical theory to the most important four isotopomers of ozone introduced above: ${ }^{16} \mathrm{O}^{16} \mathrm{O}^{18} \mathrm{O}$, ${ }^{16} \mathrm{O}^{18} \mathrm{O}^{16} \mathrm{O},{ }^{16} \mathrm{O}^{18} \mathrm{O}^{18} \mathrm{O}$, and ${ }^{18} \mathrm{O}^{16} \mathrm{O}^{18} \mathrm{O}$. Calculations are carried out within a dimensionally reduced (approximate) model of $\mathrm{O}_{3} .{ }^{31}$ The main goal is to obtain some insight into the process (2), stabilization of scattering resonances $\mathrm{O}_{3}^{*}$ by collision with Ar, where, according to Marcus's hypothesis, the $\eta$-effect might originate from.

\section{THEORETICAL FRAMEWORK}

\section{A. Reaction mechanism}

Kinetics of the processes (1)-(2) is described within micro-canonical framework, where different scattering resonances of $\mathrm{O}_{3}^{*}$ are treated as different chemical species. ${ }^{32-34}$ For each scattering resonance $\mathrm{O}_{3}^{(i)}$ at energy $E_{i}$ the processes affecting its population $\left[\mathrm{O}_{3}^{(i)}\right]$ are considered and the corresponding rate constants are introduced. Those are as follows.

(i) Formation of $\mathrm{O}_{3}^{(i)}$ from isotopically labeled reagents characterized by the second-order rate coefficient $k_{i}^{\text {form }}$

$$
\mathrm{O}_{2}+\mathrm{O} \rightarrow \mathrm{O}_{3}^{(i)}
$$

(ii) Spontaneous unimolecular decay of $\mathrm{O}_{3}^{(i)}$ onto products characterized by the first-order rate coefficient $k_{i}^{\mathrm{dec}}$

$$
\mathrm{O}_{3}^{(i)} \rightarrow \mathrm{O}_{2}+\mathrm{O} .
$$

(iii) Stabilization of $\mathrm{O}_{3}^{(i)}$ by collision with bath gas particle $\mathrm{M}$ characterized by the second-order rate coefficient $k_{i}^{\text {stab }}$

$$
\mathrm{O}_{3}^{(i)}+\mathrm{M} \rightarrow \mathrm{O}_{3}+\mathrm{M}^{*}
$$

(iv) Collision-induced dissociation $\mathrm{O}_{3}^{(i)}$ onto oxygen atoms and molecules characterized by the second-order rate coefficient $k_{i}^{\text {diss }}$

$$
\mathrm{O}_{3}^{(i)}+\mathrm{M}^{*} \rightarrow \mathrm{O}_{2}+\mathrm{O}+\mathrm{M} .
$$

The coefficients $k_{i}^{\text {form }}$ and $k_{i}^{\text {dec }}$ for each scattering resonance are related to each other through the micro-canonical equilibrium constant: ${ }^{28} k_{i}^{\text {form }}=k_{i}^{\text {dec }} K_{\text {eq }}\left(E_{i}\right)$. This equilibrium constant for each scattering resonance is computed statistically, using known formula. ${ }^{28}$ All other moieties are obtained from quantum mechanics. Namely, the width $\Gamma_{i}$ of quantum scattering resonance $\mathrm{O}_{3}^{(i)}$ gives us directly the value of $k_{i}^{\mathrm{dec}}=\Gamma_{i}$. The values of $k_{i}^{\mathrm{stab}}$ and $k_{i}^{\mathrm{diss}}$ are computed from corresponding cross sections in the $\mathrm{O}_{3}^{(i)}+\mathrm{M}$ collision dynamics simulations. One important difference from our previous work ${ }^{28}$ is that we do not employ the low-pressure assumption anymore and do not neglect the finite decay rates. Instead, similar to analysis of experimental recombination kinetics, ${ }^{9-14}$ we assume the steady-state conditions for concentration of each state $\mathrm{O}_{3}^{(i)}$, which allows deriving analytic 
expression for the third-order recombination rate coefficient of the overall reaction, ${ }^{30}$

$$
\kappa^{\mathrm{rec}}=\sum_{i} \frac{\Gamma_{i} K_{\mathrm{eq}}\left(E_{i}\right)}{\Gamma_{i}+\left(k_{i}^{\mathrm{stab}}+k_{i}^{\mathrm{diss}}\right)[\mathrm{M}]} k_{i}^{\mathrm{stab}},
$$

where the sum is over all scattering resonances $\mathrm{O}_{3}^{(i)}$.

Note that in our model of kinetics we neglect back excitations, $\mathrm{O}_{3}+\mathrm{M}^{*} \rightarrow \mathrm{O}_{3}^{(i)}+\mathrm{M}$, assuming that concentration of formed ozone $\left[\mathrm{O}_{3}\right]$ is small, and transitions between different scattering resonances, $\mathrm{O}_{3}^{(i)}+\mathrm{M} \rightarrow \mathrm{O}_{3}^{(j)}+\mathrm{M}$, assuming that their populations are entirely determined by equilibrium with reagent and product combinations of $\mathrm{O}_{2}+\mathrm{O}$. At low and moderate pressures of the bath gas $[\mathrm{M}]$ these processes are less important than those discussed above.

It is instructive to express the rate coefficient $k_{i}^{\text {stab }}$ through cross section $\sigma_{i}^{\text {stab }}$, and the equilibrium constant $K_{\text {eq }}\left(E_{i}\right)$ through the ratio of relevant partition functions. This allows rewriting Eq. (8) in the following transparent form: ${ }^{28}$

$$
\kappa^{\mathrm{rec}}=C \sum_{i} w_{i} \sigma_{i}^{\mathrm{stab}},
$$

in order to emphasize that the recombination rate coefficient is computed as a weighted sum of stabilization cross sections $\sigma_{i}^{\text {stab }}$ over all scattering resonances. The temperature- and pressure-dependent unit-less weight $w_{i}$ for each resonance is given by

$$
w_{i}=\frac{\Gamma_{i}(2 J+1) e^{-E_{i} / k T}}{\Gamma_{i}+\left(k_{i}^{\text {stab }}+k_{i}^{\text {diss }}\right)[\mathrm{M}]} .
$$

The factor $C$, common for all resonances and introduced for convenience, is

$$
C=\left(\frac{8 k T}{\pi \mu}\right)^{1 / 2} \frac{1}{Q_{\mathrm{el}} Q_{\mathrm{tr}} Q_{\mathrm{rot}}} .
$$

Here $\mu$ is the reduced mass for collision of $\mathrm{Ar}+\mathrm{O}_{3}$. The partition functions $Q_{\mathrm{el}}, Q_{\mathrm{tr}}$, and $Q_{\mathrm{rot}}$ of the reactant/product $\mathrm{O}_{2}$ in Eq. (11) are computed statistically. ${ }^{28}$

\section{B. Adiabatic bending model of ozone}

Quantum dynamics calculations presented in this paper were carried out within the dimensionally reduced model of $\mathrm{O}_{3}$ in which the bond distances $R_{1}$ and $R_{2}$ were treated explicitly (two most relevant vibrational degrees of freedom), while the bending motion of $\mathrm{O}_{3}$ was restricted to the ground vibrational state. ${ }^{26-28}$ The first step of this procedure is minimization of three-dimensional potential energy surface (PES) of ozone along the bending coordinate, for each set of fixed values of $R_{1}$ and $R_{2} \cdot{ }^{31}$ This procedure gives a relaxed two-dimensional PES, $V_{\text {pot }}\left(R_{1}, R_{2}\right)$. A contour line of this surface is given in Fig. 1, which exhibits the covalent well of $\mathrm{O}_{3}$ connected to two $\mathrm{O}+\mathrm{O}_{2}$ entrance/exit channels. Schrodinger equation (time-independent for the spectra and time-dependent for the collision dynamics) is solved on this surface by expanding the vibrational wave function $\Psi\left(R_{1}, R_{2}\right)$ over the two-dimensional grid of points, optimized to the shape of the surface, and using the FFT technique to compute the action of kinetic energy operator $\hat{T}_{\text {kin }}$ onto $\Psi\left(R_{1}, R_{2}\right)$. Further details of our approach can be found in Refs. 26-28.

In such dimensionally reduced model the excited bending states of $\mathrm{O}_{3}$ are missing, so the density of states is lower compared to true ozone molecule, roughly, by a factor of seven. This simplifies the problem and makes calculations of $\mathrm{O}_{3}^{*}+\mathrm{M}$ collision dynamics computationally affordable. The retained vibrational states describe stretching of bonds in $\mathrm{O}_{3}$. They include not only two normal-mode progressions of states-the symmetric-stretch and the asymmetric-stretch states but also two local-mode progressions of states, found closer to dissociation threshold and associated with $\mathrm{O} \cdots \mathrm{OO}$ and $\mathrm{OO} \cdots \mathrm{O}$ vibrations. Examples of wave functions for such states, for the case of the doubly substituted isotopomer, are given in Fig. 1. Nodal structure of the normal mode wavefunction in Fig. 1(a) follows the symmetric-stretch coordinate $\left(R_{1}+R_{2}\right) / \sqrt{2}$, while the nodal structure of the local mode wave-function in Fig. 1(b) follows the bond-stretch coordinate $R_{2}$. Although the dimensionally reduced model does not offer a complete description of the ozone forming reaction, still, it allows obtaining new and valuable insight into the stabilization process (2). Detailed tests of this model were reported in our earlier publication (Sec. IV A on Page 12 of Ref. 26), using the PES of Babikov et al. ${ }^{35}$

\section{Mixed quantum/classical dynamics}

Dynamics of the ozone-forming reaction is treated using the mixed quantum/classical theory for collisional energy

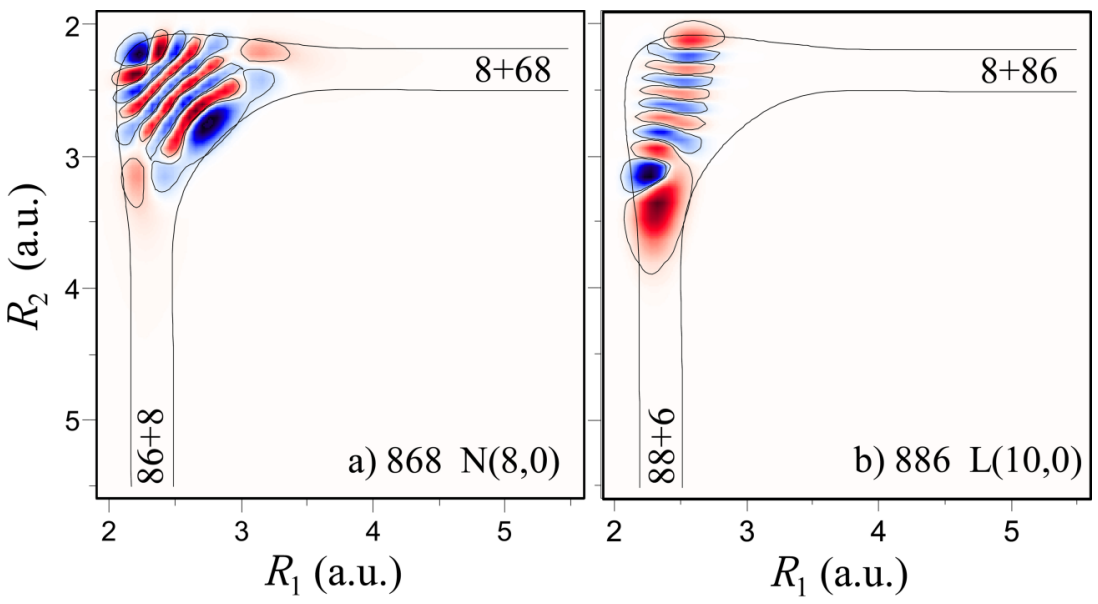

FIG. 1. Relaxed 2D PES of ozone used in this work. Contours are given to emphasize two formation/decay channel of the recombination reaction. Channels are labeled by the corresponding reagents $\mathrm{O}_{2}+\mathrm{O}$ producing either (a) symmetric ${ }^{18} \mathrm{O}^{16} \mathrm{O}^{18} \mathrm{O}$, or (b) asymmetric ${ }^{18} \mathrm{O}^{18} \mathrm{O}^{16} \mathrm{O}$. Typical vibrational wave functions for a normal mode state $\mathrm{N}(8,0)$ and a local mode state $\mathrm{L}(0,10)$ in the dimensionally reduced model of ozone are also shown. 
transfer and ro-vibrational energy flow developed earlier. All details of this method can be found in Refs. 26-28. Here we give a brief summary and emphasize new features, relevant to the isotope effect. The vibrational motion of $\mathrm{O}_{3}^{*}$ is treated using the time-dependent Schrödinger equation and the wave-packet technique. This allows capturing quantum effects associated with molecular vibration, such as zero-point energy, quantization of states, tunneling, scattering resonances, and symmetry. At the same time, the advantage is taken of the quasi-classical regime usually valid for rotational and translational degrees of freedom at room temperature. Namely, the collisional $\mathrm{O}_{3}^{*}+\mathrm{M}$ motion responsible for scattering is described by classical trajectories. The rotational motion of $\mathrm{O}_{3}^{*}$ is also treated classically within the fluid rotor approach. ${ }^{26,36,37}$ The rotation-vibration interaction in $\mathrm{O}_{3}^{*}$ is included by introducing the centrifugal potential term $V_{\text {rot }}$ into the vibrational Hamiltonian

$\hat{H}=\hat{T}_{\text {kin }}+V_{\text {pot }}\left(R_{1}, R_{2}\right)+V_{\text {rot }}\left(R_{1}, R_{2}\right)+V_{\text {opt }}\left(R_{1}, R_{2}\right)$.

This term represents rotational energy of the molecule and is a continuous smooth function of its shape, i.e., the function of its internal vibrational coordinates $R_{1}$ and $R_{2}$. This approach is expected to work better than any other method of angular momentum decoupling, since the tensor of inertia of the molecule is not fixed at a single chosen molecular configuration, but changes smoothly as molecular shape is distorted by evolution of vibrational wave function $\Psi\left(R_{1}, R_{2}\right)$. We compute $V_{\text {rot }}\left(R_{1}, R_{2}\right)$ numerically on the same grid of points used to propagate the vibrational wave packet $\Psi\left(R_{1}, R_{2}\right)$. In this way, the rotational motion of the molecule, described classically, affects its vibrational motion, treated with quantum mechanics. The energy is exchanged between translational, rotational, and vibrational degrees of freedom, but the total energy is conserved. ${ }^{26,36,37}$ The sudden collision approximation is avoided, while rotation of $\mathrm{O}_{3}^{*}$ (including rotational quenching/excitation by collisions with $\mathrm{M}$ ) is treated explicitly in a broad range of $J$ values, here up to $J \sim 90$.

Finally, the complex absorbing potential $V_{\text {opt }}\left(R_{1}, R_{2}\right)$ in the following analytic form: ${ }^{38}$

$V_{\mathrm{opt}}\left(R_{1}, R_{2}\right)=\left\{\begin{array}{cl}0, & R \leq R_{c}, \\ -i A_{c} \exp \left[-W_{c} /\left(R-R_{c}\right)\right], & R>R_{c},\end{array}\right.$

where $R=\sqrt{R_{1}^{2}+R_{1}^{2}}$ is introduced into the asymptotic part of the PES in order to compute energies $E_{i}$ and width $\Gamma_{i}$ of scattering resonances $\mathrm{O}_{3}^{(i)}$ as complex eigenvalues $E_{i}-i\left(\Gamma_{i} / 2\right)$, which correspond to complex eigenfunctions $\Phi_{i}\left(R_{1}, R_{2}\right)$. Based on a rigorous convergence study, the following set of potential parameters was chosen: $R_{c}=7 a_{0}$, $W_{c}=6 a_{0}$, and $A_{c}=10^{4} \mathrm{~cm}^{-1}$.

\section{State-to-state transitions}

In the mixed quantum-classical method the probabilities of vibrational state-to-state transitions, for each collision trajectory, are obtained by projecting the final wave packet $\Psi\left(R_{1}, R_{2}\right)$ onto the vibrational eigenstates $\Phi_{j}\left(R_{1}, R_{2}\right)$ of the final rotational state,

$$
p_{j, i}=\left|\left\langle\Phi_{j}\left(R_{1}, R_{2}\right) \mid \Psi\left(R_{1}, R_{2}\right)\right\rangle\right|^{2} .
$$

Index $i$ labels initial states $\mathrm{O}_{3}^{(i)}$ and means that at the initial moment of time (before the $\mathrm{O}_{3}^{*}+\mathrm{M}$ collision) the wave packet was started in a given vibrational eigenstate $\Phi_{i}\left(R_{1}, R_{2}\right)$. Transition probabilities $p_{j, i}$ can be used to define stabilization probability for each initial vibrational state as

$$
P_{i}^{\mathrm{stab}}=\sum_{j}^{E<0} p_{j, i} .
$$

Note that summation in Eq. (14) is over the final vibrational states at energies below dissociation threshold, $E_{j}<0$, i.e., over all stable states of ozone. This is a very natural definition of stabilization, where the processes of rotational quenching/excitation of the molecule are taken into account. Generally, the final rotational state of the molecule (after collision) is different from the initial rotational state (before collision), due to rotational energy transfer going on simultaneously with vibrational energy transfer. Equation (14) is another new feature of this work, different from the previous work, ${ }^{28}$ where the purely vibrational quenching was regarded as stabilization, without taking into account the rotational energy transfer. It appears, however, that rotational energy loss is the main stabilization pathway.

Probability of dissociation is computed as

$$
P_{i}^{\mathrm{diss}}=1-\sum_{j} p_{j, i},
$$

where the sum is over all bound states and narrow scattering resonances. Cross sections are obtained from probabilities $P_{i}^{\text {stab }}$ and $P_{i}^{\text {diss }}$ as average over the collision trajectories. Namely, for stabilization, we use

$$
\sigma_{i}^{\text {stab }}=\pi b_{\max }^{2} \frac{\sum_{\text {traj }} b P_{i}^{\text {stab }}}{\sum_{\text {traj }} b},
$$

and similar for dissociation. Note that the sum over trajectories includes sampling over the thermal distribution of $\mathrm{O}_{3}^{*}+\mathrm{M}$ collision energies. Furthermore, the number of trajectories for different rotational states, within given vibrational state, are different and proportional to their weights $w_{i}$. Convergence is checked with respect to the values of $\kappa_{i}^{\text {rec }}$ that give contribution of each vibrational state into the overall recombination process: $\kappa^{\mathrm{rec}}=\sum_{v i b} \kappa_{i}^{\mathrm{rec}}$. In this formula, summation is over vibrational states only. The rotational states are sampled as initial classical conditions for each vibrational state, and are summed into $\kappa_{i}^{\text {rec }}=C \sum_{\text {rot }} w_{i} \sigma_{i}^{\text {stab }}$, including their weights. This sampling procedure is explained in detail in Ref. 28.

\section{RESULTS AND ANALYSIS}

\section{A. Energies and widths of resonances}

Wave functions $\Phi_{i}\left(R_{1}, R_{2}\right)$, energies $E_{i}$ and widths $\Gamma_{i}$ were computed for symmetric and asymmetric isotopomers of singly and doubly substituted ozone isotopologues: ${ }^{16} \mathrm{O}^{18} \mathrm{O}^{16} \mathrm{O},{ }^{16} \mathrm{O}^{16} \mathrm{O}^{18} \mathrm{O},{ }^{18} \mathrm{O}^{16} \mathrm{O}{ }^{18} \mathrm{O}$, and ${ }^{18} \mathrm{O}^{18} \mathrm{O}^{16} \mathrm{O}$, within the dimensionally reduced model discussed above, for 
$0 \leq J \leq 90,|K| \leq J$. The values of energies for $J=0$ are given in Table I, together with state assignments in terms of the normal $\mathrm{N}\left(v_{1}, v_{2}\right)$ or local $\mathrm{L}\left(v_{1}, v_{2}\right)$ vibration mode quantum numbers. For the normal mode states the values of $v_{1}$ and $v_{2}$ designate symmetric and asymmetric stretch quanta, respectively, while for the local mode states they give the number of quanta along $R_{1}$ and $R_{2}$ (see Fig. 1). For each isotopomer in Table I, fourteen upper vibrational states, most relevant to the recombination process, are included (out of 51 states in ${ }^{16} \mathrm{O}^{18} \mathrm{O}^{16} \mathrm{O},{ }^{16} \mathrm{O}^{16} \mathrm{O}^{18} \mathrm{O}$, and ${ }^{18} \mathrm{O}^{16} \mathrm{O}^{18} \mathrm{O}$, and 54 states in ${ }^{18} \mathrm{O}^{18} \mathrm{O}^{16} \mathrm{O}$ ). These states exhibit up to eleven quanta of vibrational excitation. At $J=0$ all these states are bound vibrational states at energies $E_{i}<0$. They become scattering resonances only when a sufficient amount of rotational excitation lifts them above the dissociation threshold $(E>0)$.

The widths of states $\Gamma_{i}$ (decay rates of resonances) change smoothly as $J$ and $K$ increase. In Fig. 2 we present several typical dependencies of $\Gamma_{i}(J, K)$. Frames (a) and (c) give examples of the normal mode states in symmetric and asymmetric isotopomers, respectively, while frame (e) corresponds to the local mode state. Color indicates the absolute value of $\Gamma_{i}$, with green corresponding roughly to the interval from $\Gamma_{i} \approx 5 \cdot 10^{-3}$ to $2 \cdot 10^{-2} \mathrm{~cm}^{-1}$. We found that for the normal mode states the width of resonance $\Gamma_{i}$ does not increase beyond $10^{-2} \mathrm{~cm}^{-1}$, even at very high levels of rotational excitation $(J, K)$. This can be rationalized by the vibrational character of these states, which is a pure symmetric stretch (e.g., $\mathrm{N}(8,0)$ in frames (a) and (c) of Fig. 2). Namely, exciting vibrations of two bonds in $\mathrm{O}_{3}$ simultaneously and in-phase does not promote dissociation onto $\mathrm{O}_{2}+\mathrm{O}$. This is reflected by increased lifetimes and relatively narrow resonance widths of these states. In contrast, for the local mode states (such as $\mathrm{L}(0,10)$ in frame (e) of Fig. 2) resonances exhibit much broader widths, up to $\Gamma_{i} \approx 2 \cdot 10 \mathrm{~cm}^{-1}$ (red color) because the vibrational character of this state correlates well with the asymptotic $\mathrm{O}_{2}+\mathrm{O}$ motion. Typically, the values of $\Gamma_{i}$ for the local mode states are significantly larger, compared to $\Gamma_{i}$ for the normal mode states, for all isotopic combinations we studied.

The corresponding weights $w_{i}$ of resonances are also presented in Fig. 2, frames (b), (d), and (f). These pictures show very clearly which values of $J$ and $K$ are important for the recombination process (red color, $w_{i} \approx 5$ and higher) and which are not (dark blue color, $w_{i}<0.05$ ). We see that at very high levels of rotational excitation $(J>60)$ the weights $w_{i}$ of resonances are small. This is due to the Boltzmann factor in Eq. (9), vanishing at high energies. At low $J$, when resonances sit deep behind the centrifugal barrier, their weights $w_{i}$ can also be small, but in such cases due to very small values of $\Gamma_{i}$ in the numerator of Eq. (9). For example, at $P=0.1$ bars the weights are negligible if the values of widths are $\Gamma_{i} \leq 10^{-5} \mathrm{~cm}^{-1}$ (dark blue color). Larger weights are obtained for broader resonances, $10^{-3} \leq \Gamma_{i} \leq 10^{-2} \mathrm{~cm}^{-1}$ (cyan to green colors), at moderate levels of rotational excitation $(20<J<60)$, at energies near the top of centrifugal barrier or slightly above it. The weights close to the maximum possible $w_{i} \approx(2 J+1) e^{-E_{i} / k T}$ are obtained for resonances with $\Gamma_{i} \sim 3 \cdot 10^{-1} \mathrm{~cm}^{-1}$ (lemon-yellow color). Typically, the values of $w_{i}$ for the local mode states are significantly higher compared to $w_{i}$ for the normal mode states.

Note also that all three examples presented in Fig. 2 belong to the doubly substituted case. Similar pictures for singly substituted isotopologue can be found in the recent paper. ${ }^{30}$ Importantly, both singly and doubly substituted isotopologues share the properties discussed above.

TABLE I. Energies, assignments, and contributions to recombination rates for upper vibrational states of symmetric and asymmetric ozone isotopomers in the dimensionally reduced model.

\begin{tabular}{|c|c|c|c|c|c|c|c|c|c|c|c|c|}
\hline$\#$ & \multicolumn{3}{|c|}{ Symmetric ${ }^{16} \mathrm{O}^{18} \mathrm{O}^{16} \mathrm{O}$} & \multicolumn{3}{|c|}{ Asymmetric ${ }^{16} \mathrm{O}^{16} \mathrm{O}^{18} \mathrm{O}$} & \multicolumn{3}{|c|}{ Symmetric ${ }^{18} \mathrm{O}^{16} \mathrm{O}^{18} \mathrm{O}$} & \multicolumn{3}{|c|}{ Asymmetric ${ }^{18} \mathrm{O}^{18} \mathrm{O}^{16} \mathrm{O}$} \\
\hline 54 & & & & & & & & & & -38.6 & $\mathrm{~L}(2,7)$ & 0.124 \\
\hline 53 & & & & & & & & & & -118.7 & $\mathrm{~L}(7,2)$ & 0.354 \\
\hline 52 & & & & & & & & & & -150.5 & $\mathrm{~L}(11,0)$ & 0.338 \\
\hline 49 & -355.1 & $\mathrm{~L}(1,8)$ & 0.433 & -315.0 & $\mathrm{~L}(10,0)$ & 0.689 & -376.6 & $\mathrm{~L}(8,1)$ & 0.304 & -467.9 & $\mathrm{~L}(0,10)$ & 0.389 \\
\hline 48 & -409.6 & $\mathrm{~N}(7,1)$ & 0.072 & -318.9 & $\mathrm{~L}(1,8)$ & 0.324 & -456.4 & $\mathrm{~L}(0,10)$ & 0.352 & -517.7 & $\mathrm{~L}(8,1)$ & 0.121 \\
\hline 47 & -456.1 & $\mathrm{~L}(10,0)$ & 0.382 & -331.0 & $\mathrm{~N}(7,1)$ & 0.111 & -456.4 & $\mathrm{~L}(10,0)$ & 0.352 & -537.6 & $\mathrm{~N}(7,1)$ & 0.051 \\
\hline 46 & -456.1 & $\mathrm{~L}(0,10)$ & 0.382 & -435.0 & $\mathrm{~L}(0,10)$ & 0.331 & -470.7 & $\mathrm{~N}(7,1)$ & 0.136 & -597.3 & $\mathrm{~L}(10,0)$ & 0.111 \\
\hline 45 & -573.9 & $\mathrm{~N}(6,2)$ & 0.278 & -466.1 & $\mathrm{~N}(6,2)$ & 0.176 & -589.2 & $\mathrm{~N}(6,2)$ & 0.122 & -688.2 & $\mathrm{~N}(6,2)$ & 0.062 \\
\hline 40 & -1080.2 & $\mathrm{~L}(7,1)$ & 0.010 & -952.4 & $\mathrm{~L}(0,9)$ & 0.006 & -1079.9 & $\mathrm{~L}(1,7)$ & 0.005 & & & \\
\hline 39 & -1080.2 & $\mathrm{~L}(1,7)$ & 0.010 & -1028.5 & $\mathrm{~L}(1,7)$ & 0.009 & -1079.9 & $\mathrm{~L}(7,1)$ & 0.005 & & & \\
\hline 38 & -1240.8 & $\mathrm{~N}(7,0)$ & 0.002 & -1196.9 & $\mathrm{~N}(7,0)$ & 0.002 & -1339.7 & $\mathrm{~N}(7,0)$ & 0.000 & & & \\
\hline & \multicolumn{2}{|c|}{ Total } & 2.228 & \multicolumn{2}{|c|}{ Total } & 2.784 & \multicolumn{2}{|c|}{ Total } & 1.724 & & Total & 2.089 \\
\hline
\end{tabular}



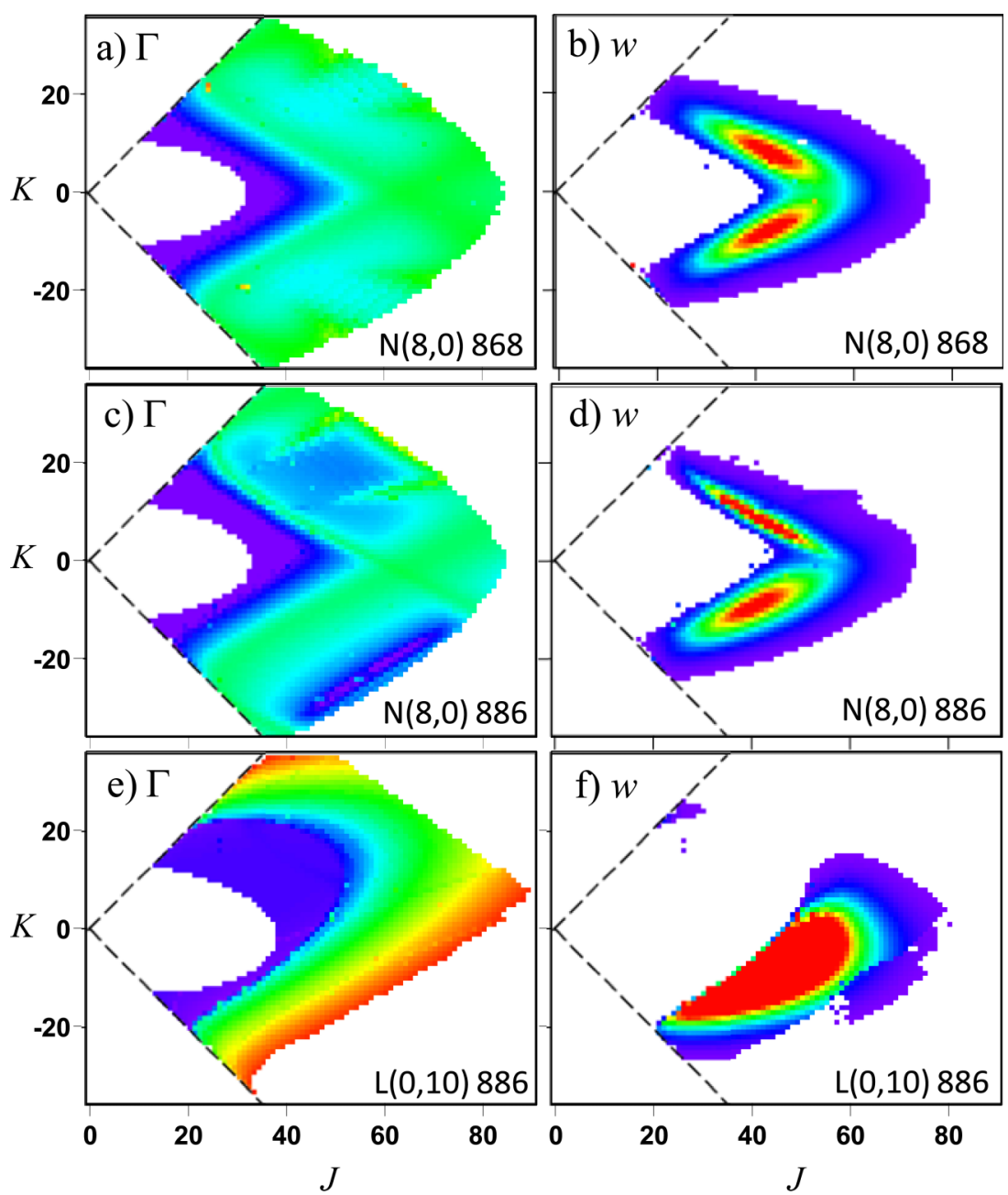

FIG. 2. Rotational distributions of resonance widths $\Gamma_{i}(J, K)$ and weights $w_{i}(J, K)$, in the left and right columns, respectively, for several typical vibrational states in the dimensionally reduced model of ozone: ((a) and (b)) The normal mode state $\mathrm{N}(8,0)$ of symmetric ${ }^{18} \mathrm{O}^{16} \mathrm{O}^{18} \mathrm{O}$; ((c) and (d)) the normal mode state $\mathrm{N}(8,0)$ of asymmetric ${ }^{18} \mathrm{O}^{18} \mathrm{O}^{16} \mathrm{O}$; ((e) and (f)) the local mode state $\mathrm{L}(0,10)$ of asymmetric ${ }^{18} \mathrm{O}^{18} \mathrm{O}^{16} \mathrm{O}$.

\section{B. Stabilization and dissociation cross sections}

Stabilization cross sections $\sigma_{i}^{\text {stab }}$, to be used in Eq. (8), were computed from the collision dynamics simulations using Eq. (16), for each ro-vibrational metastable state $\mathrm{O}_{3}^{(i)}$. Note that for each vibrational state of Table I there are hundreds of relevant rotational states (see Fig. 2), and the amount of data produced in our calculations is huge. In order to make sense of all these data we tried to analyze how the computed values of $\sigma_{i}^{\text {stab }}$ depend on various characteristics of the ro-vibrational states. For example, we found that there is a reasonable correlation between the total energy of the scattering resonance $E_{i}$, and the stabilization cross section $\sigma_{i}^{\text {stab }}$. We analyzed these $\sigma_{i}^{\text {stab }}\left(E_{i}\right)$ dependencies for the states of different character (normal vs. local vibration modes), for symmetric vs. asymmetric isotopomers, and for singly vs. doubly substituted isotopologues. Figure 3 summarizes our findings. Each frame of this figure combines data for all ro-vibrational states in Table I, of all characters (both local and normal modes together) of both singly and doubly substituted isotopologues. The only difference is that Fig. 3(a) contains data for symmetric isotopomers only, while Fig. 3(b) contains the same data for asymmetric isotopomers. In this way, we can test the old hypothesis that asymmetric isotopomers of ozone are stabilized at a higher rate compared to symmetric isotopomers. ${ }^{29,30}$
Visually, the data of Figs. 3(a) and 3(b) are very similar. To quantify the differences (if any) between stabilization cross section for symmetric and asymmetric isotopomers, we tried to fit each of the distributions by a rather typical double-exponential model of energy transfer ${ }^{28}$

$$
\sigma^{\text {stab }}(E)=\sigma_{0}^{\text {stab }}\left(\exp \left\{-\frac{E}{\gamma}\right\}+c \exp \left\{-\frac{E}{d}\right\}\right) .
$$

The values of fitting coefficients are given in Table II. The corresponding curves are plotted on top of Figs. 3(a) and 3(b), and together in Fig. 3(c). Clearly, there is a very little difference between them, within $7 \%$, while the statistical error of our calculations for each case is close to $4 \%$. Importantly, we do not see that the resonance states of asymmetric ozone are stabilized more efficiently. In fact, we see that stabilization cross sections of resonances in symmetric molecules are a bit higher (just opposite to what we expected to find), but this difference is small and is not really meaningful, since it is within statistical error of sampling.

The third curve in Fig. 3(c) represents a fit of all data, symmetric and asymmetric together, i.e., of the data in Figs. 3(a) and 3(b) combined. The fitting coefficients for this case are also given in Table II. This common fit is very similar to the individual fits for two symmetries, which strongly supports the following conclusion: We see no isotope 

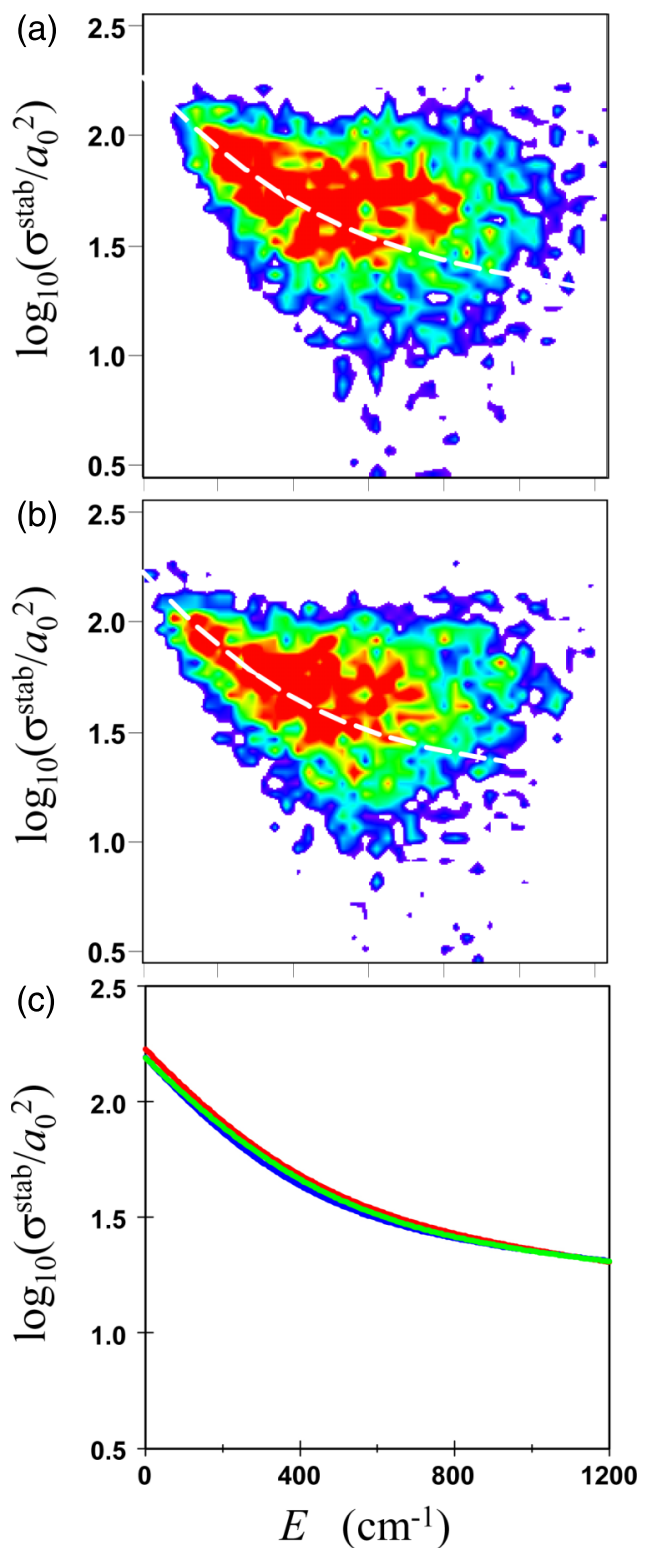

FIG. 3. Distribution of stabilization cross sections for scattering resonances in (a) symmetric and (b) asymmetric isotopomers of ozone. Frame (c) gives three fits by the double-exponential model: one for each distribution (red for symmetric and blue for asymmetric) and the common fit of all data together (green).

effect in stabilization cross sections that could potentially lead to a significant and robust $\eta$-effect.

To build a better model for energy transfer we introduced, in addition to the double-exponential dependence on total resonance energy $E_{i}$, the dependence on vibrational energy of the resonance $E_{i}^{\mathrm{vib}}$, expressed in the following way:

TABLE II. Fitting parameters for analytic dependence of Eq. (17) for stabilization cross section.

\begin{tabular}{lcccc}
\hline \hline & $\sigma_{0}^{\text {stab }}\left(a_{0}^{2}\right)$ & $\gamma\left(\mathrm{cm}^{-1}\right)$ & $d\left(\mathrm{~cm}^{-1}\right)$ & $c$ \\
\hline Symmetric & 131.60 & 202.58 & 1968.72 & 0.2778 \\
Asymmetric & 122.39 & 193.88 & 2523.20 & 0.2653 \\
Common & 123.92 & 218.40 & 2881.77 & 0.2430 \\
\hline \hline
\end{tabular}

$$
\begin{aligned}
\sigma^{\text {stab }}\left(E, E^{\mathrm{vib}}\right)= & \sigma_{0}^{\text {stab }}\left(\exp \left\{-\frac{E}{\gamma}\right\}+c \exp \left\{-\frac{E}{d}\right\}\right) \\
& \times\left(1-\frac{A_{\mathrm{v}}}{1+\left(E^{\mathrm{vib}} / k T\right)^{2}}\right) .
\end{aligned}
$$

For this model, for the fit of both symmetric and asymmetric isotopomers together (i.e., common fit of all the data we obtained), the values of fitting parameters are $\sigma_{0}^{\text {stab }}$ $=184.46 a_{0}^{2}, \gamma=177.41 \mathrm{~cm}^{-1}, d=1462.92 \mathrm{~cm}^{-1}, c=0.2881$, and $A_{\mathrm{v}}=0.9939$. Overall, the quality of this analytic model is very reasonable. For example, if we replace the actual computed values of $\sigma_{i}^{\text {stab }}\left(E_{i}\right)$ for each ro-vibrational state by those obtained from the Eq. (18), the final value of the recombination rate coefficient $\kappa^{\text {rec }}$ in Eq. (8) changes only by $+5.6 \%,+2.7 \%,+4.6 \%$, and $+2.2 \%$ in the cases of ${ }^{16} \mathrm{O}^{18} \mathrm{O}^{16} \mathrm{O}$, ${ }^{16} \mathrm{O}^{16} \mathrm{O}^{18} \mathrm{O},{ }^{18} \mathrm{O}^{16} \mathrm{O}^{18} \mathrm{O}$, and ${ }^{18} \mathrm{O}^{18} \mathrm{O}^{16} \mathrm{O}$, respectively.

Following the same lines, the dissociation cross sections for both isotopomers of both isotopologues were fit together by the following analytic expression:

$$
\sigma^{\text {diss }}(E)=\sigma_{0}^{\text {diss }}\left(1+\tanh \left\{\frac{\left(E-E_{0}^{\text {diss }}\right)}{\gamma^{\text {diss }}}\right\}\right) .
$$

This fit was done separately for the local and normal modes, because the local modes dissociate easier and exhibit larger dissociation cross sections. ${ }^{28}$ The values of fitting parameters are $\sigma_{0}^{\text {diss }}=9.59 a_{0}^{2}, \gamma^{\text {diss }}=480 \mathrm{~cm}^{-1}$, and $E_{0}^{\text {diss }}=651 \mathrm{~cm}^{-1}$ for the normal modes and $\sigma_{0}^{\text {diss }}=46.76 a_{0}^{2}, \gamma^{\text {diss }}=239 \mathrm{~cm}^{-1}$, and $E_{0}^{\text {diss }}=544 \mathrm{~cm}^{-1}$ for the local mode states. The quality of analytic model (19) is very reasonable. For example, if we replace the actual computed values of $\sigma_{i}^{\text {diss }}\left(E_{i}\right)$ for each ro-vibrational state by those obtained from expression (19), the final value of the recombination rate coefficient $\kappa^{\text {rec }}$ in Eq. (8) changes only by $-0.1 \%,+0.2 \%,-1.0 \%$, and $-1.5 \%$ in the cases of ${ }^{16} \mathrm{O}^{18} \mathrm{O}^{16} \mathrm{O},{ }^{16} \mathrm{O}^{16} \mathrm{O}^{18} \mathrm{O},{ }^{18} \mathrm{O}^{16} \mathrm{O}^{18} \mathrm{O}$, and ${ }^{18} \mathrm{O}^{18} \mathrm{O}^{16} \mathrm{O}$, respectively.

If we use two models together, namely, if we replace the actual computed values of both $\sigma_{i}^{\text {stab }}\left(E_{i}\right)$ and $\sigma_{i}^{\text {diss }}\left(E_{i}\right)$ for each ro-vibrational state by those obtained from the Eqs. (18) and (19), the final value of the recombination rate coefficient $\kappa^{\text {rec }}$ in Eq. (8) changes only by $+5.2 \%,+2.9 \%,+2.8 \%$, and $-0.3 \%$ in the cases of ${ }^{16} \mathrm{O}^{18} \mathrm{O}^{16} \mathrm{O},{ }^{16} \mathrm{O}^{16} \mathrm{O}^{18} \mathrm{O},{ }^{18} \mathrm{O}^{16} \mathrm{O}^{18} \mathrm{O}$, and ${ }^{18} \mathrm{O}^{18} \mathrm{O}^{16} \mathrm{O}$, respectively.

\section{Discussion}

The analytic models derived above for stabilization and dissociation cross sections of ozone resonances represent the most important practical result of this paper. Very little information about efficiency of collisional stabilization is available from literature and, to our best knowledge, no information at all about the collision-induced dissociation cross sections for ozone. One experimental datum we are aware of is the average value of energy transfer $\langle\Delta E\rangle$ $=18 \mathrm{~cm}^{-1}$ derived by Troe ${ }^{39}$ for ${ }^{16} \mathrm{O}^{16} \mathrm{O}^{16} \mathrm{O}$, which is not directly relevant to the absolute value of stabilization cross sections for resonances. It is probably true to say that theoretical progress on modelling the recombination reaction that forms ozone was hindered, mostly, by the 
absence of virtually any information on stabilization step (2). Further confusion/complication came from a widely accepted hypothesis that stabilization cross sections for symmetric and asymmetric isotopomers of ozone may be significantly different and would be the source of the mysterious $\eta$-effect. In contradiction with this hypothesis, we found no significant difference in stabilization and dissociation cross sections for symmetric and asymmetric isotopomers. The analytic models we propose here, Eqs. (18) and (19), are applicable to both symmetric and asymmetric isotopomers (with the same set of fitting coefficients) and provide a very reasonable description of the process. They can be used in conjunction with more accurate models of the ozone molecule itself, such as the full-dimensional 3D model of $\mathrm{O}_{3},{ }^{40}$ which would be the major improvement over the dimensionally reduced simplified 2D ozone model employed here.

Although we do not see any appreciable difference in stabilization cross sections for symmetric and asymmetric isotopomers, we see significant differences in recombination rate coefficients, which looks very much like the $\eta$-effect. Namely, the last row of Table I gives the total recombination rate coefficient, summed over all vibrational states. For asymmetric isotopomer the rate coefficient is higher than that for symmetric isotopomer, by $25 \%$ and $21 \%$ in the singly and doubly substituted cases, respectively. These numbers can be compared with experimental data, but indirectly, as explained below.

Consider a singly substituted asymmetric product ${ }^{16} \mathrm{O}^{16} \mathrm{O}^{18} \mathrm{O}$. It can be formed from two entrance channels of the PES that correspond to two distinct reactant combinations: ${ }^{16} \mathrm{O}+{ }^{16} \mathrm{O}^{18} \mathrm{O}$ and ${ }^{16} \mathrm{O}^{16} \mathrm{O}+{ }^{18} \mathrm{O}$. In the experiment ${ }^{13}$ the rate coefficients for these processes are 1.45 and 0.92 (relative to the rate coefficient of the usual ${ }^{16} \mathrm{O}+{ }^{16} \mathrm{O}^{16} \mathrm{O} \rightarrow{ }^{16} \mathrm{O}^{16} \mathrm{O}^{18} \mathrm{O}$ ). Likewise, the symmetric product ${ }^{16} \mathrm{O}^{18} \mathrm{O}^{16} \mathrm{O}$ can be formed from two channels, but those are equivalent: ${ }^{16} \mathrm{O}+{ }^{18} \mathrm{O}{ }^{16} \mathrm{O}$ and ${ }^{16} \mathrm{O}^{18} \mathrm{O}+{ }^{16} \mathrm{O}$, and the experimental rate coefficient for each is 1.08 (relative to the same Ref. 13). Thus, the ratio of total formation rates of ${ }^{16} \mathrm{O}^{16} \mathrm{O}^{18} \mathrm{O}$ to ${ }^{16} \mathrm{O}^{18} \mathrm{O}^{16} \mathrm{O}$ should be computed as $(1.45+0.92) /(1.08+1.08)=1.10$, which means that it is $10 \%$ higher for asymmetric isotopomer, compared to the symmetric one. Similar calculations for the doubly substituted case, ${ }^{18} \mathrm{O}^{18} \mathrm{O}^{16} \mathrm{O}$ vs. ${ }^{18} \mathrm{O}^{16} \mathrm{O}{ }^{18} \mathrm{O}$, using experimental data of Ref. 13, give $(0.92+1.50) /(1.04$ $+1.04)=1.16$. Thus, asymmetric ${ }^{18} \mathrm{O}^{18} \mathrm{O}^{16} \mathrm{O}$ is formed at a rate that is $16 \%$ higher, compared to the corresponding symmetric isotopomer ${ }^{18} \mathrm{O}^{16} \mathrm{O}^{18} \mathrm{O}$. These experimental numbers, $10 \%$ and $16 \%$, are somewhat smaller than the isotope effect that we see in our calculations: $25 \%$ and $21 \%$ for the singly and doubly substituted cases, respectively.

However, it should be pointed out that mechanistic interpretation of experimental data is not straightforward. It should probably be taken into account that a recent analysis by Troe $^{39}$ indicates that at room temperature the contribution of energy transfer mechanism, Eqs. (1) and (2), is only 40\% of the total rate, with almost $\sim 60 \%$ coming from the chaperon mechanism of recombination that, usually, is not associated with any anomalous isotope effects. ${ }^{41}$ This information can be taken into account by adding to our computed rates of the energy transfer mechanism (Table I) a contribution of the chaperon mechanism that is isotopomer independent, with the estimated rate computed as 6/4 of the energytransfer rate, averaged over four considered isotopomers: $\kappa=6 / 4 \times 2.207 \times 10^{-35}=3.310 \times 10^{-35}\left(\mathrm{~cm}^{6} / \mathrm{s}\right)$. Obviously, this would be a rough estimate. With such adjustment the computed rate coefficient for asymmetric isotopomer is higher than that for symmetric isotopomer by $10 \%$ and $8 \%$ in the singly and doubly substituted cases, respectively. This is somewhat closer to the experimentally observed differences $\left(10 \%\right.$ and $16 \%$, respectively $\left.{ }^{13}\right)$ but this agreement should not be overemphasized, due to roughness of the adjustment made, and also due to the reasons explained below.

First of all, the absolute values of recombination rate coefficients in our calculations are, roughly, one order of magnitude smaller compared to experimental values. ${ }^{13,39}$ This is due to employment of the dimensionally reduced 2D model, where the excited bending states of $\mathrm{O}_{3}$ are missing, and the density of states is much smaller. The second problem, also due to employment of the dimensionally reduced $2 \mathrm{D}$ model, is that the differences between ${ }^{16} \mathrm{O}^{18} \mathrm{O}^{16} \mathrm{O},{ }^{16} \mathrm{O}^{16} \mathrm{O}^{18} \mathrm{O}$, ${ }^{18} \mathrm{O}^{16} \mathrm{O}^{18} \mathrm{O}$, and ${ }^{18} \mathrm{O}^{18} \mathrm{O}^{16} \mathrm{O}$ are artificially exaggerated, since the number of states is small (only upper fourteen vibrational states contribute, see Table I). In such situation the difference in the properties of one state in symmetric and asymmetric isotopomers, such as energy, or vibrational character, often has large influence, and the isotope effect, to some extent, is accidental. For example, from Table I, we see that although all four considered ozone molecules have the same number of the normal mode states (with slightly different energies), the isotopomer ${ }^{18} \mathrm{O}^{18} \mathrm{O}^{16} \mathrm{O}$ has three more local mode states (\#52, 53 , and 54, see Table I) that are not present in ${ }^{16} \mathrm{O}^{18} \mathrm{O}^{16} \mathrm{O}$, ${ }^{16} \mathrm{O}^{16} \mathrm{O}^{18} \mathrm{O}$, or ${ }^{18} \mathrm{O}^{16} \mathrm{O}^{18} \mathrm{O}$. This is because only one isotopomer ${ }^{18} \mathrm{O}^{18} \mathrm{O}^{16} \mathrm{O}$ has two heavy isotopes in the same bond, which produces the local vibrations of ${ }^{18} \mathrm{O}^{18} \mathrm{O}$ moiety, characterized by lower frequency and more states. Also, one can spot in Table I that the upper normal mode state $\mathrm{N}(8,0)$, which is \#51, is characterized by significantly lower energies in the doubly substituted cases ${ }^{18} \mathrm{O}^{16} \mathrm{O}^{18} \mathrm{O}$ and ${ }^{18} \mathrm{O}^{18} \mathrm{O}^{16} \mathrm{O}$, compared to the singly substituted ${ }^{16} \mathrm{O}^{18} \mathrm{O}^{16} \mathrm{O}$ and ${ }^{16} \mathrm{O}^{16} \mathrm{O}^{18} \mathrm{O}$, which also reduced significantly its contribution to the recombination process. Such significant differences are not expected to occur between isotopomers or isotopologues of the real ozone molecule, where the density of states is higher (roughly by a factor of seven), and evolution of one state can't make a big difference.

We can point out that the $\eta$-effect we observe in our calculations comes from widths $\Gamma_{i}$ (or decay rates) of scattering resonances that tend to be larger in asymmetric ozone molecules than in their symmetric isotopomers ${ }^{30}$ which translates into larger weights $w_{i}$ of resonances, and finally into larger recombination rates for asymmetric ozone molecules. This effect was discussed in detail in our recent paper ${ }^{30}$ and is also consistent with recent work on atom-exchange process in $\mathrm{O}_{2}+\mathrm{O}$ collisions, where significant isotope effects were observed and attributed to scattering resonances in $\mathrm{O}_{3}$ alone, without any bath gas involved. ${ }^{42-45}$ However, for rigorous description of this effect in symmetric and asymmetric isotopomers of both singly and doubly substituted isotopologues, the dimensionally reduced $2 \mathrm{D}$ model of $\mathrm{O}_{3}$ 
is insufficient. It appears to be too rough. More accurate full-dimensional 3D description of ozone molecule is still needed.

It is also important to note that a new PES for ozone is now available from the recent work of Dawes and coworkers. ${ }^{46-48}$ It has a flat shoulder in the channels region, instead of a reef. This surface already allowed to improve temperature dependence of the atom-exchange rates in $\mathrm{O}_{2}+\mathrm{O}$ collisions $^{43-45}$ and may help to resolve some of the issues discussed above.

\section{CONCLUSIONS}

In this paper we presented the mixed quantum/classical calculations of the energy transfer mechanism for the recombination reaction that forms ozone, within the dimensionally reduced 2D model of ozone molecule, with bending motion neglected. Calculations were carried out at room temperature for symmetric and asymmetric isotopomers of singly and doubly substituted isotopologues: ${ }^{16} \mathrm{O}^{18} \mathrm{O}^{16} \mathrm{O}$, ${ }^{16} \mathrm{O}^{16} \mathrm{O}^{18} \mathrm{O},{ }^{18} \mathrm{O}^{16} \mathrm{O}^{18} \mathrm{O}$, and ${ }^{18} \mathrm{O}^{18} \mathrm{O}^{16} \mathrm{O}$. The processes of resonance formation, decay, collisional dissociation, and stabilization were all characterized and taken into account. The focus was on the stabilization step where, according to the well accepted hypothesis, the isotopic $\eta$-effect might originate from. We found no difference in cross sections of stabilization of scattering resonances in symmetric and asymmetric isotopomers. We also derived simple and general analytic models for stabilization and dissociation cross sections that can be applied to resonances in any ozone molecule (symmetric or asymmetric, singly or doubly substituted). This model will be used in the future studies of the ozone forming reaction, where the full-dimensional accurate $3 \mathrm{D}$ model for ozone vibrations will be employed. Present calculations show some isotope effect, that looks similar to the experimentally observed $\eta$-effect, and the origin of this phenomenon is in formation/decay rates of scattering resonances, determined by their widths, that are somewhat larger in symmetric isotopomers than in their symmetric analogues. However, the present two-dimensional model is too rough and incapable of describing consistently all the features of the isotopic effects in ozone. Calculations using an accurate $3 \mathrm{D}$ model, ${ }^{40}$ and a new PES of Dawes, ${ }^{46-48}$ are ongoing and will be reported elsewhere.

\section{ACKNOWLEDGMENTS}

This research was supported by the NSF Atmospheric Chemistry Program, Division of Atmospheric Sciences, Grant No. AGS-1252486. This research used resources of the National Energy Research Scientific Computing Center, which is supported by the Office of Science of the U.S. Department of Energy under Contract No. DE-AC02-05CH11231.
${ }^{1}$ K. Mauersberger, Geophys. Res. Lett. 8, 935, doi:10.1029/GL008i008p00935 (1981).

${ }^{2}$ K. Mauersberger, B. Erbacher, D. Krankowsky, J. Guenther, and R. Nickel, Science 283, 370 (1999).

${ }^{3}$ M. H. Thiemens and J. E. Heidenreich iii, Science 219, 1073 (1983).

${ }^{4}$ J. E. Heidenreich iii and M. H. Thiemens, J. Chem. Phys. 84, 2129 (1986).

${ }^{5}$ M. H. Thiemens, Science 283, 341 (1999).

${ }^{6} \mathrm{M}$. Thiemens, in Treatise on Geochemistry (Elsevier, 2007), pp. 1-24.

${ }^{7}$ M. H. Thiemens, Annu. Rev. Earth Planet. Sci. 34, 217 (2006).

${ }^{8}$ M. Thiemens, S. Chakraborty, and G. Domingues, Annu. Rev. Phys. Chem. 63, 155 (2012).

${ }^{9}$ D. Krankowsky and K. Mauersberger, Science 274, 1324 (1996).

${ }^{10}$ S. M. Anderson, D. Hulsebusch, and K. Mauersberger, J. Chem. Phys. 107, 5385 (1997)

${ }^{11}$ C. Janssen, J. Guenther, D. Krankowsky, and K. Mauersberger, J. Chem. Phys. 111, 7179 (1999).

${ }^{12}$ S. Wolf, M. Bitter, D. Krankowsky, and K. Mauersberger, J. Chem. Phys. 113, 2684 (2000).

${ }^{13}$ C. Janssen, J. Guenther, K. Mauersberger, and D. Krankowsky, Phys. Chem. Chem. Phys. 3, 4718 (2001).

${ }^{14}$ B. Tuzson, "Symmetry specific study of ozone isotopomer formation," Doctoral Dissertation (University of Heidelberg, 2005).

${ }^{15}$ Y. Q. Gao and R. A. Marcus, Science 293, 259 (2001).

${ }^{16}$ Y. Q. Gao and R. A. Marcus, J. Chem. Phys. 116, 137 (2002).

${ }^{17}$ Y. Q. Gao, W.-C. Chen, and R. A. Marcus, J. Chem. Phys. 117, 1536 (2002).

${ }^{18}$ B. C. Hathorn and R. A. Marcus, J. Chem. Phys. 111, 4087 (1999).

${ }^{19}$ B. C. Hathorn and R. A. Marcus, J. Chem. Phys. 113, 9497 (2001).

${ }^{20}$ M. Kryvohuz and R. A. Marcus, J. Chem. Phys. 132, 224305 (2010).

${ }^{21}$ N. Ghaderi and R. Marcus, J. Phys. Chem. B 115, 5625 (2011); J. Phys. Chem. A 118, 10166 (2014).

${ }^{22}$ D. Charlo and D. C. Clary, J. Chem. Phys. 117, 1660 (2002).

${ }^{23}$ D. Charlo and D. C. Clary, J. Chem. Phys. 120, 2700 (2004).

${ }^{24}$ T. Xie and J. M. Bowman, Chem. Phys. Lett. 412, 131 (2005).

${ }^{25}$ M. V. Ivanov and R. Schinke, Mol. Phys. 108, 259 (2010).

${ }^{26}$ M. V. Ivanov and D. Babikov, J. Chem. Phys. 134, 144107 (2011).

${ }^{27}$ M. V. Ivanov and D. Babikov, J. Chem. Phys. 134, 174308 (2011).

${ }^{28}$ M. V. Ivanov and D. Babikov, J. Chem. Phys. 136, 184304 (2012).

${ }^{29}$ M. V. Ivanov and D. Babikov, Chem. Phys. Lett. 535, 173 (2012).

${ }^{30}$ M. V. Ivanov and D. Babikov, Proc. Natl. Acad. Sci. U. S. A. 110, 17708 (2013)

${ }^{31}$ L. Jiang and D. Babikov, Chem. Phys. Lett. 474, 273 (2009).

${ }^{32}$ D. Babikov, B. Kendrick, R. B. Walker, and R. T Pack, J. Chem. Phys. 119, 2577 (2003).

${ }^{33}$ R. T Pack, R. B. Walker, and B. K. Kendrick, J. Chem. Phys. 109, 6714 (1998).

${ }^{34}$ S. Y. Grebenshchikov and R. Schinke, J. Chem. Phys. 131, 181103 (2009).

${ }^{35}$ D. Babikov, B. Kendrick, R. B. Walker, R. T Pack, P. Fleurat-Lesard, and R. Schinke, J. Chem. Phys. 118, 6298 (2003).

${ }^{36}$ A. Semenov and D. Babikov, J. Chem. Phys. 138, 164110 (2013).

${ }^{37}$ A. Semenov, M. Ivanov, and D. Babikov, J. Chem. Phys. 139, 074306 (2013).

${ }^{38}$ Numerical Grid Methods and their Application to Schrodinger Equation, edited by C. Cerjan (Kluwer, 1993), pp. 195-205.

${ }^{39}$ K. Luther, K. Oum, and J. Troe, Phys. Chem. Chem. Phys. 7, 2764 (2005).

${ }^{40}$ A. Teplukhin and D. Babikov, J. Chem. Educ. 92, 305 (2015).

${ }^{41}$ M. V. Ivanov and R. Schinke, J. Chem. Phys. 124, 104303 (2006).

${ }^{42}$ Z. Sun, L. Liu, S. Y. Lin, R. Schinke, H. Guo, and D. H. Zhang, Proc. Natl. Acad. Sci. U. S. A. 107, 555 (2010).

${ }^{43}$ Y. Li, Z. Sun, B. Jiang, D. Xie, R. Dawes, and H. Guo, J. Chem. Phys. 141, 081102 (2014).

${ }^{44}$ W. Xie, L. Liu, Z. Sun, H. Guo, and R. Dawes, J. Chem. Phys. 142, 064308 (2015).

${ }^{45}$ Z. Sun, D. Yu, W. Xie, J. Hou, R. Dawes, and H. Guo, J. Chem. Phys. 142, 174312 (2015).

${ }^{46}$ R. Dawes, P. Lolur, J. Ma, and H. Guo, J. Chem. Phys. 135, 081102 (2011).

${ }^{47}$ R. Dawes, P. Lolur, A. Li, B. Jiang, and H. Guo, J. Chem. Phys. 139, 201103 (2013).

${ }^{48}$ S. Ndengué, R. Dawes, X.-G. Wang, T. Carrington, Z. Sun, and H. Guo, J. Chem. Phys. 144, 074302 (2016). 